\title{
Discussion on "Primary Standard of Light." Atlantic City, N. J., July 2, 1908
}

A. E. Kennelly (by letter): The paper presents a certain fundamental physical proposition relating to a fundamental standard of luminous intensity, and also a practical proposal suggesting a means for carrying out the proposition. In so far as concerns the physical proposition, it seems to be unassailable, and to offer a theoretical solution for the problem of realizing a standard luminous intensity of definite physiological value, and definitely measured energy; that is, in ergs per steradian, or ergs per cubic centimeter from point to point of the luminous beam.

As regards the practical suggestion of carrying this method into effect, it seems to me a great deal of experimental work will be necessary to develop the method. It would be necessary not only to develop the bolometric method of measuring the energy in the three component beams with a satisfactory degree of accuracy for standard purposes, but it would also be necessary to determine the physiological stimulus of each of these three colors throughout the range of intensity required in photometric measurements. This is for the reason that in order to measure the light given by any particular lamp, such as a Hefner flame, for example, it would be necessary to arrive at a color balance on the photometer, by altering the ratio of the intensities in the three component beams. This would involve an alteration in the luminous stimulus, or light sensation, of unit intensity of the composite light so produced, and the luminous intensity of the Hefner flame could only be expressed in terms of standard light after taking into account the change in physiological effect of the altered components.

Nevertheless, Dr. Steinmetz's proposal is definite, and offers a definite chance of realizing a true determination of light in terms of energy, as the outcome of experimental research along three defined wave lengths or luminous frequencies.

The suggestion in the paper that the scale of abscissas should be logarithmic, instead of numerical, seems to be excellent for many graphical purposes. According to this suggestion, the visible spectrum would be divided into three larger regions (violet, green, and red) and four smaller regions, or "semitones," namely, indigo and blue, on one side of the green, and yellow and orange on the other. This would make an optical scale of approximately three tones and four semi-tones, or ten semi-tones altogether. The division of the spectrum would thus naturally fall into decimal subdivisions instead of into Angström units. Each first decimal place, or tenth root of 2, would correspond approximately to a semi-tone of color, while two such decimal divisions would correspond approximately to a tone of color.

Edwin P. Hyde (by letter): It would be very desirable indeed, both from theoretical and practical considerations, to be able to define our standard of light in terms of the C. G. S. 
system of units, rather than have if without any known connection with other physical quantities. The suggestion of Dr. Steinmetz would seem to make possible such a definition. According to the suggestion we would define, not a standard of luminous intensity, but a standard of luminous flux; and we would measure the intensity of an unknown source by comparing the illumination produced by the flux from that source with the illumination produced by the standard flux. Since according to our present methods what we actually do is to compare illuminations and reason by means of known or assumed laws to the luminous intensities of the sources producing the illuminations, it is only necessary that we have a standard luminous flux, and not that we have a source of standard luminous intensity.

The principal difficulty to be overcome in realizing such a standard as Dr. Steinmetz suggests is in accomplishing with the requisite accuracy the absolute measurement of the small amount of energy contained in a single bright line in the visible spectrum. The determination of the proper proportions of energy in the three regions of the spectrum to give the required color, and the reproduction of these three colors in their proper proportions, could probably be accomplished with sufficient accuracy, but the determination of the energy of each region in absolute units would be a much more difficult undertaking, It would seem to me that a suggestive method of procedure might consist of: first, in obtaining as intense radiation as possible for the objective measurements, and then cutting down the intensity, if necessary, by sectored disks or other suitable means for the photometric measurements; and secondly, in measuring objectively the energy both of the component wave-lengths and of the combined flux-the former to give the proper proportions, and the latter to give the total luminous flux. By this method, slight errors in determining the proper proportions of the component parts would only enter as second order effects if the total flux is measured accurately; and the larger the total quantity of energy measured objectively the more accurately can the measurement be made when we are dealing with relatively small quantities of energy.

Although there would be numerous advantages in such a standard as Dr. Steinmetz proposes, if such a standard could be realized, there is one slight disadvantageous feature, which, however, is not serious. It has usually been customary to take the standard of white light as the standard also of spectral distribution in spectrophotometric measurements. This can only be done, of course, when the standard has either a continuous spectrum, or else a large number of bright lines well distributed in the visible region of the spectrum. The use for spectrophotometric measurements of such a standard as that proposed by Dr. Steinmetz would not seem feasible.

With the adoption of a common international unit of $\mathrm{lu}$ - 
minous intensity maintained arbitrarily by means of incandescent lamp secondary standards, such as has been proposed, the demand for a suitable primary standard of light is not as urgent as it has been heretofore; it is nevertheless just as important as ever, and the proposal of Dr. Steinmetz to define the standard in watts, with a prescribed spectral distribution, is very suggestive.

W. S. Franklin (by letter): The establishment of a reliable and reproducible light standard is of very great importance, and we naturally look to our Bureau of Standards to carry out such researches as may be necessary for its realization. I therefore move that the Bureau of Standards be requested by the Institute of Electrical Engineers to undertake such researches as may be necessary to determine the practicability of the light standard suggested by Dr. Steinmetz, ${ }^{1}$ and, to develop such a primary standard of light, if it be found practicable.

In asking the Institute to place itself on record in this matter, I must say that, as it seems to me, Dr. Steinmetz's proposal cannot be a complete solution of the problem. Indeed, I do not believe the problem is capable of complete solution. Our present ideas of precision, and the theory of errors of observation as it stands at present, have been developed almost wholly in connection with extremely precise measurements, such as measurements in astronomy and geodesy, where the thing which is measured approximates very closely an actual "state of permanency," that is, where the thing which is measured actually exists as an unvarying physical condition. In attempting to establish a standard of light, however; it is important, as it seems to me, to understand that the present ideas of precision of measurement are in many cases inapplicable. Measurement in its most general aspects consists in bringing two things or systems into a kind of congruence, and all errors of observation originate in the incompleteness of this congruence test. In view of this general statement it is possible to distinguish two classes of errors of observation, namely, (a) errors which are due to erratic variations in the thing or system which is being measured, and (b) errors which are due to erratic variations in the measuring devices. The former being inherent in the thing which is measured, may be called intrinsic errors; and the latter, being outside of the thing which is measured, may be called extrinsic errors.

The probable error of a result, in so far as it depends upon extrinsic errors (irregularities in the measuring devices), is a measure of precision in the ordinary sense of that term, whereas the probable error of a result, in so far as it depends upon intrinsic errors (irregularities in the thing or system which is being measured), is a measure of the degree of indefiniteness of the thing which is measured.

1. Proceedings American Institute Electrical Engineers, March, 1908, p. 297. 
It is generally possible to distinguish between extrinsic errors and intrinsic errors, and it is desirable to use the term probable error (as calculated from extrinsic errors) as a measure of precision, and to use the term probable departure for that part of the probable error, ordinarily so-called, which depends upon intrinsic errors.

In order to make these ideas perfectly clear, let us consider a particular case of measurement relating to erratically ${ }^{2}$ varying conditions. For example, let us consider the repeated measurement of the current which is delivered by an electric railway power station. The successive measured values of the current differ greatly from each other, and one might proceed in the ordinary way in the determination of the "probable error" of the result. Suppose, however, that the ammeter is carefully studied by measuring with it a very carefully controlled current output which is kept as nearly constant as possible. In this way the systematic and erratic errors of the ammeter may be determined, and the probable error of an ammeter reading under these conditions would be a measure of the precision of the instrument. Let $a$ be the probable error of a single observation of the ammeter, so determined. Let $A$ be the probable error of a single reading of the ammeter as determined from the residuals of the observed values of the current output of the station. Then $A^{2}-a^{2}$ is the probable departure of the current output of the station at any given instant from its mean value; that is to say, the difference between the mean value of the current output of the station and its actual value at any instant is as likely to be greater than $A^{2}-a^{2}$ as it is to be less than $A^{2}-a^{2}$.

Suppose the value of $a$ in the above discussion to be one ampere and the value of $A$ to be ten amperes. Then it is evident that the extrinsic errors (due to irregularities in the measuring device) in the above measurement are entirely negligible in their effect on the result as compared with the intrinsic errors (due to irregularities in the system which is being studied).

Ordinary photometry presents a case in which intrinsic errors are very great, because the actual intensity of light sensation depends upon so many varying conditions which are beyond control; in fact, the intensity of the light sensation which is produced by a given beam of light depends upon manifold individual peculiarities of the person, upon the degree of fatigue of the retina, and upon the degree to which the sensation is enhanced by attention, to say nothing of variations in composition of the light (relative intensities of the various wave-lengths which

2. Any set of varying conditions must be thought of as erratic when no attempt is made to analyze the varying conditions into systematic groups. Thus the variation of current output of an electric railway power station is to be thought of as erratic unless an attempt is made to study the mean daily and hourly fluctuations of load. For the present purpose, the current output of such a station is assumed to be erratic. 
are present). It would therefore seem to be hopeless, and indeed meaningless, to attempt to establish a unit of light which would be completely definite except in so far as the physical character of the light is concerned. The proposal of Dr. Steinmetz does, in fact, seem to provide for complete definiteness of the light unit from a physical point of view, and the degree of precision with which this light unit might be reproduced would probably be so great as to leave nothing to be desired, considering the very great intrinsic errors in photometric measurements.

Carl Hering: I have been very much interested in this paper by Dr. Steinmetz, and am very glad to see a suggestion in a practical form of a new unit of light, based on the power equivalent. It interests me personally, because I have for a number of years (since 1901 or 1902) recommended that our unit of light should be based on the power unit, that is, that the unit of light should be defined as the light produced by one erg per second converted entirely into light.* But I suggested no specific way in which that could be carried out practically other than to base it on one selected wave-length, or on a certain average wave-length, and I am therefore very much pleased to see that Dr. Steinmetz has offered this very interesting and apparently possible solution.

There is another reason why it is desirable to use the power unit in which to express our unit of light. We have at present three groups of units, the absolute (or electrical) units, the gravitational units, and the heat units. All of our units, except those of light, can be referred to one of these three. They have as connecting links the mechancial equivalent of heat, the acceleration of gravity, and the specific heat of water. Each of these three groups of units was originally started on an entirely independent basis, and the connecting links had to be derived afterwards by experiment. We have at present a unit of light which does not belong to any of these; it is based on an entirely new foundation, and it will be necessary to find by experiment the connecting links with these three groups of units. Dr. Steinmetz's suggestion is not to form such a new group, but to start by making the unit of light coincident with one of the existing groups, namely, the electrical or absolute units.

The term "unit of light", used throughout Dr. Steinmetz's paper, might be confused with the term "candle-power" by those who have not paid particular attention to the subject of light units. Of course, he does not mean candle-power, he means flux of light, as he stated in his abstract. There is evidently no possibility of an equivalent between watts and candle-power. The only equivalent can be between watts and flux of light. Flux of light is equal to candle-power multiplied by a solid angle, or to illumination multiplied by a surface. $\dagger$ We use the term

\footnotetext{
* See Conversion Tables, Hering, p. 3; also p. 147.

$\uparrow$ See Conversion Tables, Hering, p. 147.
} 
"watts per candle-power"--but, of course, that is well known to be an irrational expression, although it has come into our nomenclature. We mean, of course, watts per spherical or hemispherical candle-power.

I call attention to an error in the diagram of the musical scale, in which the "a " to the left should be "e." I suggest that the English equivalents be added to this musical scale, as it will then be more easily understood by English readers; Dr. Steinmetz has apparently adopted the German nomenclature. Why does he reverse the conventional methods in the diagram by having the shorter wave-length to the left and the longer wave-length to the right, instead of the reverse, which is the universal method in musical practice; also in showing the spectrum, in which, I believe, it is conventional to show the red end to the left and the blue end to the right? There is an error in the text a little further on: one tone represents 60 deg., not 30 deg.

At the close of the paper, Dr. Steinmetz says that any color can be made up of these three. I am interested in knowing whether he is really quite sure of that, for the following reason: If we take three colored pigments and mix them together, we will get a certain color; now if we take some of that mixed pigment, and mix with it some white pigment, and then take some more and mix with it some black pigment, we will have, with the original mixture, three distinct colors. Each one of them will have the same relative quantities of the three original colored pigments in it, and yet it will in itself be an entirely different color from the other two. It might be said that they are shades of the same color. Now the question is, whether a parallel to this exists in the mixing of light rays. That is, is there in the mixing of light rays, something which corresponds to the admixture of white pigment or black pigment to a colored pigment?

Some years ago, when I was interested in this subject of the equivalent between power units and light units, I desired to know about what the magnitude of that unit would probably be; that is, what the conversion factor between light and power would be. I found some determinations made quite a number of years ago and after recalculating them to bring them to our present units, I found the figures to be 5.3 spherical candles per watt.* That figure might be termed the electrical equivalent of light. Hence, if the original tests were correct (I took them as $I$ found them and am not responsible for their correctness), the figure will be about of that magnitude, which corresponds to 0.188 watt per spherical candle-power. If this figure be correct, that would be the ultimatum which we will ever be able to reach, as it would mean an efficiency of 100 per cent.

* Mechanical Equivalent of Light, Hering, Elec. World, April 20, 1901, p. 631 . 
The unit which Dr. Steinmetz proposes naturally involves the solid angle when reduced to candle-power, and we therefore have the choice now in defining that unit, of selecting either the steradian (or absolute unit solid angle) or the sphere, as the solid angle to be included in that unit. Selecting the sphere eliminates the troublesome $4 \pi$ from most of our calculations of light flux, which are made in spherical candles, and are not based on steradians.

Clayton H. Sharp: I would like to submit that when any member of this Institute is going to present to us a general solution of the electric circuit and the general solution of the perhaps equally difficult problem of the standard of light, he should at least give us a chance to catch our breath in between.

It looks to me as if this were, in a way, a general solution of this problem. The whole question of the standards of light, the history of the subject, shows how difficult the problem has been. For many years the flame standards were used, and were sufficiently good for the purpose. With the introduction of electric lamps, which were capable of more accurate measurement, a greater precision in photometry was at once demanded, and certain fundamental difficulties which exist in flame standards became known for the first time.

As a solution of the question of a more reliable standard of light, Violle came to the front with his propositions, namely, the use, as a standard, of the light emitted by a square centimeter of platinum at its point of solidification. This proposal, being on the face of it very attractive, was received and adopted at several electrical congresses, but it has never been found feasible to put it into practical operation, because a sufficiently accurate reproduction of this so-called " absolute " unit of light could not be carried out. Other propositions have been made of a similar nature, and the proposal to use energy measurements is not a new one. We find that Lummer made an extensive investigation of a standard of light which was defined in this way: "the standard light shall consist of the luminous radiation from a surface of platinum brought to such a temperature that the ratio of the radiation which it will transmit through a certain cell containing water to its total radiation per unit surface is as 1 to 10." Now, it should be theoretically possible to define a standard in this way. The great difficulty with such a definition, however, is that it involves the measurement of the ratio of the intensities of radiation, one of which, at least, is very low. Another difficulty with it is that it depends upon the radiating properties of a certain substance, namely, platinum. This latter difficulty might be obviated by using, instead of the platinum surface, a surface of a theoretical black body. However, the first difficulty would remain, and it seems to be of sufficient importance to preclude the possibility of establishing an accurate standard in this way. 
The proposition which is made to us to-day appears to be the very first one which offers a chance of the establishment of a standard of light based on energy measurements, and independent of the properties of any substance whatever. Not only does it offer us a standard of light, but it gives us also the possibility of a standard for the measurement of colored light, since a variation of the proportions of the different colors used will produce a variation in the resultant tint. The latter feature may very well be one of considerable importance in view of the ever-increasing diversity in tints of the lights that we have actually to measure in the photometer room.

As Dr. Steinmetz has said, we have to deal here with a physiological effect, and not with a purely physical effect. Ordinarily, the physiological effect which we get from an incandescent lamp, or from most of our light sources, is an effect made up of the various wave-lengths of the spectrum; in other words, the spectra of our common light sources are continuous spectra, and have difference in color on account of a continuous difference in the ratio of the intensities of the various wave-lengths.

It is to my mind an open question, one which must be submitted to experimental determination, whether we shall get ultimately the same effect from the admixture of three monochromatic lights that we would get from a polychromatic source; whether it is not possible that minor differences in the sensibility of individual eyes to the different colors will not become much more apparent when we measure a polychromatic source against a source which is made up of three colors, than when we are measuring two polychromatic sources, one against the other. Will the physiological effect of three colors mixed together in all cases be the same as the physiological effect of all the colors mixed together, though in varying proportion?

In regard to the difficulties of the actual energy measurements which are involved in the realization of this solution of the standard of light, it seems to me, while they are very considerable, yet they are not necessarily insuperable, because it is not necessary, as I understand it, to deal with any extremely small quantities of energy. There is no reason why the sources which are used for the production of these three lines should not be sufficiently powerful so that there will be no small concentration of energy in each of the lines, and so that it will become physically possible to measure the radiation-intensity of each of these lines with a sufficient degree of accuracy for the purpose. I think there is no doubt that the method ought to be given a very thorough trial indeed, and I think also that the place where it ought to be tried and investigated is the Bureau of Standards, at Washington, where the answer to the question can be found, if it can be found anywhere.

I think there was some confusion in the statements of the last speaker in regard to the nomenclature of this matter. As 
I understand it, Dr. Steinmetz's proposal looks to the production of a standard illumination on a given surface. Now, illumination is not the same thing as candle-power, though it can be referred to the theoretical point-source of light placed at a certain distance from the surface which is illuminated. Neither is illumination simply flux of light, but it is flux of light per unit area, or flux density, and consequently the proposition is to produce, really, a standard illumination on a surface, and if we wish to refer that to an imaginary point-source of light, which really does not exist in the experiment, we can say that illumination is produced by a source of one candle-power. However, the solid angle does not enter into the question directly, neither necessarily does the candle-power enter in to the question directly, nor does the $4 \pi$ come in. It is a question simply of producing a unit or standard illumination on a given surface, or unit flux density, or unit flux per unit area; for when we get right down to the fundamentals, it is flux we have to deal with in the case of light. The candle-power notion or concept is more or less fictitious and superfluous, though convenient for many purposes. For other purposes, the candlepower notion cannot enter in; we must come to the idea of luminous flux and base our computation of illumination on the idea of a luminous flux, as is quite evirlent. when we consider any space illuminated by the so-called indirect method, where the ceiling is brightly illuminated, and, serving as a secondary source of light, illuminates other parts of the room. Evidently, there is no candle-power there, it is all a case of luminous flux from the ceiling falling on other surfaces of the room and illuminating them.

I think we are to be congratulated that this first, and certainly we hope the true, solution for the absolute definition of a standard of light, emanates from this country, and is presented for the first time to this Institute.

C. A. Perkins: It is not necessary for me to add my congratulations to Dr. Steinmetz for his valuable paper, but I wish to emphasize the fact that it is a step toward the solution of a problem which is impossible from its nature.

As Dr. Steinmetz has just explained, illumination is a physiological phenomenon and cannot be measured. It is impossible to say that a red light is as bright as a blue light. It is impossible to say that a Welsbach burner is so many times as bright as a Hefner lamp. The ratio depends on the eye of the observer, and also is a matter of personal estimate or judgment, and is not capable of measurement.

Yet the proposed standard simplifies the problem of com= paring lights of different colors very materially, by reducing all the colors to three.

The next step will be to fix upon numbers which may repre, sent the brightness of each of these primary sources (in candle- 
power per watt) based upon the average judgment of a large number of observers. The number thus determined will be more or less arbitrary but will represent in a fairly satisfactory way the average judgment and the normal eye. When the relative brightness of the primary sources has been fixed upon, the various secondary standards-Hefner, pentane or incandescent lamps-may be expressed definitely in terms of the primary standards, and the comparison of colored sources will be definite, though it cannot be absolute.

John B. Taylor: Dr. Steinmetz has several times told me that he had no proper musical training or appreciation, but in this paper he gives us a rainbow set to music, and leads me to hope that he has taken up a new subject, old as an art, but still having many unsolved physical and mathematical problems.

I wish to point out a few discrepancies as they appeal to me. In the first place, if we are going to replot the spectrum on a rational basis, why do we use wave-length at all? The essence of light, as well as of sound, is frequency, and not wave-length. Sodium light has a certain rate of vibration, while its wave-length is only incidental to the transmitting medium, having one value in air and other values in glass, water, etc. The same is true of sound. Pitch depends on frequency, while the wave-length of a sound of given pitch has different values in air, hydiogen, water, and wood. So it seems we should rightly las out the spectrum on the basis of frequency instead of wave-length, frequency being really the essence of light and color.

I agree with Mr. Hering, that the English and American names of the musical tones would be more appropriate for this Institute. The German " cis's " and "fis's" are not so familiar to most of us as c-sharp and f-sharp, which in addition to the misprints and backward arrangement, have obscured, the optical and acoustical comparison. The use of frequencies, rather than wave-lengths, will naturally bring red on the familiar left end of the spectrum and the musical scale will read from left to right.

I do not see the arguments for a logarithmic scale in representing the spectrum. The musical system, both melodic and harmonic, is based entirely on the ratios of frequencies, and music as written is essentially a curve or curves with time as abscissas and logarithms of frequencies as ordinates. I have never yet been able to find that there is anything in the optical scale corresponding to this matter of ratios in the acoustical scale. It is true that certain combinations of colors will be harmonious while other combinations will not be pleasing. For example, it is generally agreed that pink and red do not go well together. Is it possible to take two different colors and say that these are not harmonious, on account of the ratio of their frequency? There is a definite period in music. In a 
well-recognized sense, after the interval of an octave, we go over the same ground again.

I hope Dr. Steinmetz can show more clearly some periodic property of the spectrum or something depending on ratios of frequencies to justify the logarithmic plot.

Carl Hering: Dr. Sharp says there is some confusion of terms in my remarks. I am confident that Dr. Steinmetz will agree with me that his proposed unit is not a unit of illumination, as Dr. Sharp says, but a unit of flux, which is equivalent to illumination multiplied by an area, or candle-power multiplied by a solid angle-either one gives the same result. I am quite familiar with the various units and measures of light and the relations between them, as I gave some attention to this question of equivalency between watts and light some years ago and I am sure there is no confusion in my mind

E. B. Rosa: I am interested in this proposition of Dr. Steinmetz, and should sincerely like to see it realized. It would be important to make a serious attempt to get a primary photometric standard in this way, which should be sufficiently satisfactory to be considered to solve the problem. It seems probable that it might be somewhat difficult to make the energy measurements with the required accuracy, and perhaps this will prove to be an insurmountable difficulty; and yet I have no doubt that if the attempt were made seriously, sufficient information and experience would result from the work to pay for the effort. even if it did not result entirely satisfactorily. It often happens that when a serious effort on a difficult problem is mare and continued, a great many new things appear in the work that are of considerable value, by-products, so to speak, of the investigation. I think this field might be a very valuable and promising one to investigate thoroughly, with the hope, of course, that the problem of finding a primary photometric standard would be solved. At any rate, I think it would be proper to consider it very carefully.

I appreciate also the words of confidence in the Bureau of Standards that have been expressed. This investigation is clearly in the field of work of the Bureau of Standards, and the Director of the Bureau would be very glad, I have no doubt, to take up the work without any request from the Institute, as soon as practicable. Nevertheless a request made by the Institute would be very proper, and I would be glad to see that request made formally, because, as you will easily understand, the Bureau has so many fundamental problems on hand, more than it can at present undertake, that it needs support in its work; it needs the expression of confidence of this Institute, and the moral support of the engineering fraternity at large in its work. Such support will enable it to get the assistance and to develop the force that it needs in order to take up such problems, and therefore it can do more of that kind of funda- 
mental work if interest is expressed in such a concrete way as is here proposed. I can easily see that such an investigation might last over a considerable period of time. We now have some problems on hand that have already extended over considerable periods of time, but in most cases they have been very profitable investigations to pursue; and entirely apart from the primary objects of the investigation, the results have been of considerable importance.

I wish again to express my interest in the suggestion of Dr. Steinmetz, and certainly consider it of value.

Carl Hering: I make the motion that the Board of Directors of this Institute be requested to refer to the Bureau of Standards the question of the establishment of a standard of light as proposed in this paper of Dr. Steinmetz. (The motion was unanimously carried.)

H. S. Carhart: I have only a few words to add to what has already been said. I suppose the reason why Dr. Steinmetz has chosen three distinct wave-lengths to enter into this combination for luminous intensity or luminous flux is the fact that there are three distinct primary color sensations. It would be desirable, first, to be assured that we get an average determination of the wave-lengths of these three primary colo1s, as applied to the eyes of different individuals, because there is individuality in this matter. Secondly, we should measure the relative energy in the three wave-lengths to produce any definite color, such as yellowish white. This task would be one of considerable difficulty. In that way we should be able to get the average energy of the primary colors required to reproduce a light of any given tint and intensity. It can readily be seen that such processes as these would necessarily require a good deal of time in order to realize them.

I think one of the speakers has been confused a little, if I may call attention to the fact-the mixture of three colored lights is a totally different thing from the mixture of pigments. The mixture of pigments is a process of absorption, and the mixture of lights is a matter of superposition and addition. If we have red glass, which cuts off the violet end of the spectrum, and blue glass, which cuts off the red end of the spectrum, and if we put one in front of the other, the only light that comes through is a little of the red. This is like the mixture of pigments. We should be very careful not to confuse mixture of pigments with mixture of colors. What we have in the paper is three colored lights, coming from three sources of definite wave-length. I am greatly interested in the proposition, and think it is really hopeful enough to suggest to the Bureau of Standards, as has already been done, for investigation.

Chas. P. Steinmetz: Three colors I selected as primary colors, since three primary colors are sufficient by their combination to produce all other colors, representing these by the ratio of the 
energies of the three primary colors. As seen, this is essentially the representation of the points of the color plane by a system of symmetrical coördinates. Combinations of two colors are not sufficient to produce all other colors, but at least three are needed. In regard to the selection of the three primary colors, I tried to get three colors forming as nearly as possible an equilateral triangle in the polar diagram, or in the color plane, as shown, so as to get the maximum accuracy in their combination. There was a second consideration. The three points of the equilateral triangle I tried to select so as to have a maximum distance from the ends of the spectrum, since at the ends of the spectrum the physiological equivalent of power is low and vanishing, and therefore if one of the lines were chosen close to the end, as for instance dark red, it would mean there would always be a large percentage, in power, of red rays, even to produce a color which appeared to the eye deficient in red. For greatest accuracy, it is however desirable to have the three numerical values of power of primary colors as nearly of the same magnitude as possible. The two outside colors, blue and red, are therefore chosen about equidistant from the ends of the spectrum, as two corners of the equilateral triangle, which brings the third corner into the middle of the spectrum, in the green. You can choose any other set of spectrum colors, but I believe the best way would be to choose the three points of an approximately equilateral triangle, with the two outside corners as far away from the ends of the spectrum as possible, as was done by me.

As regards the scale adopted, I must apologize, for my musical education was so badly neglected that I still used the Italian scale, instead of the flats and sharps of the English notation. I believe, however, it will be equally intelligible.

As regards the wave-length; what I give is not really the logarithm of the wave-length, but the logarithm of the frequency. The wave-length of light is not constant, but a function of the medium. However, the logarithm of the wavelength in any medium is the same, and is also the logarithm of the frequency, except with reversed sign and shifted decimal point. It therefore is immaterial, in the logarithmic scale, whether wave-length or frequency is used; either gives the same curve or figure, except turned over, and it obviously is immaterial whether the red end of the spectrum is on the right or on the left side.

In regard to the bearing of the acoustic scale, you will see if you look at the polar diagram, that it is material, and that the wave-length is not the right measure. In the acoustic or polar diagram, two diametrically opposite points are of complementary color, that is, combined give white light, or approximately white light (after all " white " is a rather in definite expression). This is not the case in the representation by wave-length, nor in 
the representation by frequency; but in either case you find that one-half of the visible spectrum represents much less variation of the physiological effect, that is, change of color, than the other half.

About color representation: If we represent colors by a combination of the three primary radiations, we still have different values for the same color, representing different color densities. For instance, a certain yellow light may be represented by: red, 50; green, 50 ; blue, 0 . The same yellow light, but of a lighter shade, may be represented by: red, 45 ; green, 45 ; blue, 10 . Still a lighter shade by: red, 40; green, 40; blue, 20; until ultimately you reach white light.

I believe that even the densest yellow light, produced from the primary colors: red, 50; green, 50; blue, 0 , is yet not the spectrum yellow; that is, not as pure yellow as the srectrum yellow, and that the spectrum yellow would be represented by something like: red, 55 ; green, 55 ; blue, -10 . This ratio: $55 \div 55 \div-10$ represents a point of the plane outside of the equilateral triangle formed by the primary-color points, and such probably is the case with the spectrum yellow and with all the spectrum colors, except the three colors selected as primaries.

Physically, this means that if we desire to compare the spectrum yellow with our three-color combination, no combination of red, green and blue can be found to equal the spectrum yellow, but that a combination of red and green can be found to equal a combination of the spectrum yellow and blue, and in this case the blue becomes subtractive. It is the same as balancing a weight by putting too much weight on the other scale, and then putting some weights on the first scale, i.e., balancing by taking some weights back. We may do the same in color representation. Theoretically, I believe all pure spectrum colors, except the three selected as primary, would be represented by combinations of the three primary colors with one of the primary radiations as negative.

As regards the mechanical equivalent of light, I do not agree with the value mentioned by Mr. Hering, 0.188 watts per candle-power, for the reason that efficiencies of arcs have been measured which give lower watts per mean spherical candle-power than that. In some powerful titanium arcs values considerably lower have been found, and in those cases the efficiency was still far below 100 per cent. Estimating the loss of power by invisible ultra-red and ultra-violet radiation, by heat conduction, heat convection, air currents, etc., the efficiency was undoubtedly below 50 per cent., probably nearer 10 per cent. I believe the approximate equivalent of white light is probably something more nearly fifty candle-power per watt-that is, 0.02 watts per spherical candle-power. I make no claim to this value, but it is merely an estimate based on my experienceit may be more or less, but I would expect the equivalent of 
white light to be approximately 50 candle-power per watt, or thereabouts. For other colors of light there would be different values. Probably in the case of the green light, the candlepower per watt would be much greater; in red light or blue light much less. It is a function of the color. 